CONSTRAINTS ON CENTRAL SOLAR CONDITIONS FROM HELIOSEISMOLOGY AND NEUTRINO COUNTS

\author{
John Faulkner \\ Lick Observatory \\ University of California \\ Santa Cruz, CA 95064 \\ U.S.A.
}

ABSTRACT. Interesting joint constraints are placed on central solar conditions by the solar neutrino detection rate and the frequency separations of high-order, low-degree p-modes of pulsation. A new form of diagnostic diagram is introduced that illustrates the difficulty in standard theories of simultaneously satisfying these joint constraints. A model employing WIMPs (= weakly interacting massive particles) appears to be the only model extant capable of fitting both kinds of data. Unambiguous identification and fitting of g-modes can provide a further test of the WIMP model provided a certain caution is exercised.

1. INTRODUCTION: SOLAR NEUTRINOS, P-MODES, AND CONVENTIONAL SOLAR MODELS

I introduce here a new kind of diagnostic diagram, which illustrates in a particularly clear manner the difficulty in simultaneously satisfying the constraints imposed on solar models by (i) the neutrino counts in Davis's ${ }^{37} \mathrm{Cl}$ detector (Cleveland et al., 1984) and (ii) the frequency separation of observed p-modes of pulsation (see Faulkner et al., 1986 for references and a discussion).

Figure 1 illustrates the central conditions found in standard solar models and their variants, as calculated by three groups. The we11known Bahcall standard model, omitted for clarity, would essentially complete a small parallelogram F, I2, S0, B. In the diagram the abscissa $\mathrm{T}_{\mathrm{c}} / \mu_{\mathrm{c}} \propto \mathrm{c}_{\mathrm{s}}^{2}$ was chosen because, with certain assumptions, the frequency separations of solar p-modes of like $\left(n+\frac{1}{2} l\right)$ indicate values for central sound speed $c_{s}$ somewhat lower than those obtained in the standard models. The shaded vertical region shows the desired range of $c_{s}$ estimated for models having small, essentially isothermal cores. The consequences of other assumptions, viewed as perturbations from a standard model, would yield analogous regions, though differing in detail. The ordinate $\mathrm{T}_{c} / \mu_{c}$ is also convenient; in terms of this variable the diffusively mixed models of Schatzman et al., (1981) move along an essentially horizontal line as diffusion is increased. With logarithmic scales, models of given central temperature lie on lines of slope unity 


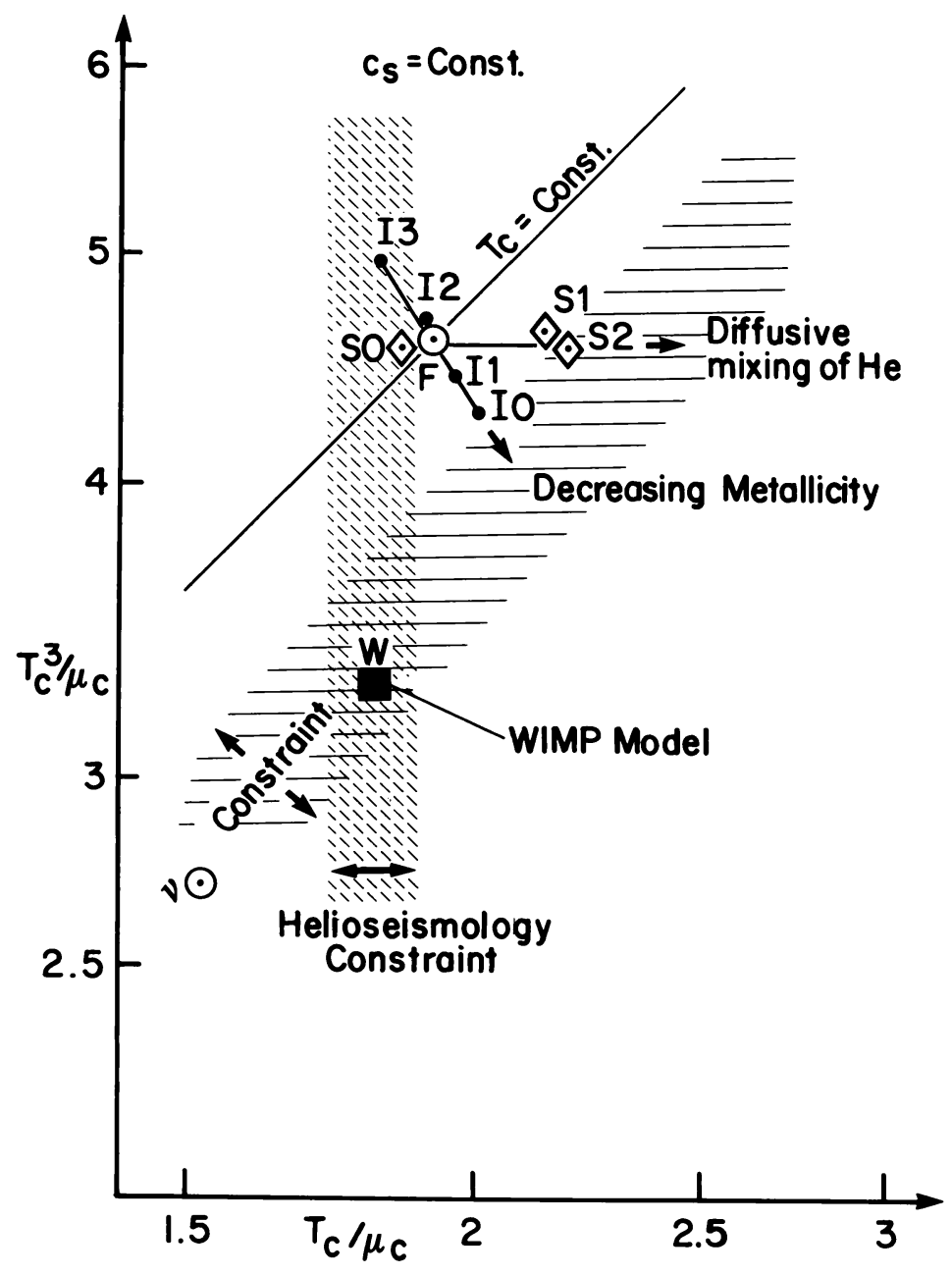

Figure 1. Central conditions for selected solar models vs. solar neutrino and helioseismology constraints. Here $\mathrm{T}_{c}$ is the temperature (in units of $10^{7} \mathrm{~K}$ ) and $\mu_{c}$ the molecular weight at the solar center; $c_{s}$ is the ${ }_{2}$ central sound speed. The abscissa $\mathrm{T}_{c} / \mu_{c} \propto c_{s}^{2}$; the ordinate $\mathrm{T}_{c} 3 / \mu_{c}$ $\propto c_{s}^{2} \mathrm{~T}_{\mathrm{c}}{ }^{2}$. Predictions of standard solar models are indicated by the heavy dot I2 (Iben, 1969 with metallicity $\mathrm{Z}=0.02$ ), circled point $\mathrm{F}$ (Faulkner and Gilliland, 1985) and the diamond point s0 (Schatzman et al., 1981 with diffusion coefficient set to zero). Points IN are Iben's metallicity variants with $\mathrm{Z}=\mathrm{N} \times 0.01$. Diamond points $\mathrm{SN}$ are diffusively mixed models by Schatzman et al., with diffusion coefficient $R_{e}^{*}=N \times 100$. Only point $W$, from the WIMP model (Faulkner and Gilliland, 1985; Faulkner, Gough and Vahia, 1986) is consistent with both helioseismology and the Davis $\nu_{\Theta}$ measurements. 
in the diagram, as drawn through point $F$, the standard model of Faulkner and Gilliland (1985).

Solutions to the solar neutrino problem require that $\mathrm{T}_{c}$ be lower than that in standard models. However, there is also a weak dependence on composition. By examining other models it was concluded that the $v_{\theta}$ constraint defined a desirable band for solution of slope 1.1 -- i.e. almost parallel to $\mathrm{T}_{\mathrm{c}}=$ constant. Two of many "conventional" attempts to resolve the solar neutrino problem are illustrated. Iben (1969) questioned, among other things, our knowledge of the solar heavy element concentration, which of course affects the opacity of solar material. However, he noted that even the reduction of $\mathrm{Z}_{\Theta}$ to zero was insufficient (as the diagram shows) to reduce the predicted $v_{0}$ count enough. Schatzman et al. suggested that mixing of the deep interior by a turbulent diffusive process would solve the problem. This re-homogenization essentially rejuvenates the solar center, returning it to conditions (lower $T_{c}, \mu_{c}$ and $\rho_{c}$ ) characteristic of a younger sun. Sufficient mixing can enable the observed $\nu_{\Theta}$ counts to be matched.

If one were concerned only to solve the solar neutrino problem, this would be all very well. However, as can be seen, the helioseismology constraint adds a wholly new dimension. One must not solve the solar neutrino problem at the expense of creating or exacerbating a solar pulsation problem! Standard models are seriously inconsistent with the solar neutrino flux, but only mildly inconsistent with the helioseismology constraint. Diffusively mixed models can solve the solar neutrino problem, but only at the unacceptable price of producing a central sound speed very much larger than indicated by helioseismology.

\section{WIMP MODELS AND P-MODES}

The WIMP model, on the other hand (Faulkner and Gilliland, 1985; Faulkner, Gough and Vahia, 1986) achieves its results in a very different way. Greatly increased central energy transport by WIMPs renders the central regions nearly isothermal and reduces the value of $T_{c} \cdot$ This in turn affects the nuclear burning history but, importantly, the slightly altered $\mu$-gradient which results is nevertheless left intact. Consequently, the central sound speed is slightly lower than in the standard model, and elsewhere the sound speed increases away from the center, as in the standard model, but for a somewhat greater distance. In contrast, in the diffusively mixed models, sound speed is not only larger at the center but also decreases throughout with radius. Thus, relative to the standard model, the behavior of sound speed perturbations in the central regions is quite opposite for the WIMP and diffusively mixed models. It was already known prior to the work of Faulkner et al. that the diffusively mixed models led to a serious mismatch with the observed p-mode frequency separations; it was the opposite character of the central perturbations found in the WIMP model which suggested and indeed led to its improved fit. 


\section{WIMP MODELS, G-MODES, AND A REPEATED WARNING}

WIMP models also differ in yet one more important way from other proposed resolutions of the solar neutrino problem. The nearly isothermal core leads to an increase in central density, in order that pressure support be maintained. Consequently (Faulkner, Gough and Vahia, 1986), the normalized asymptotic g-mode period separation (here called $P_{0}$ for consistency with Cox's summary in this volume) should be $P_{0} \sim 29-30$ minutes, rather than the $\sim 36-37$ minutes of standard models, or much larger stil1, up to $\sim 56$ minutes for the diffusively mixed models. At this meeting Fröhlich has reported that a value for $P_{0}$ of $\sim 29.8$ minutes indeed gives the best fit to ACRIM data in the frequency range from 10 to $40 \mu \mathrm{Hz}$, although the method of demonstration may leave room for doubt.

One thing is clear: an unambiguous determination of $\mathrm{P}_{0}$ for the $\mathrm{g}-$ modes should certainly distinguish the WIMP model from all others, provided one is in asymptotia. Cox, in his summary, has quite correctly warned us that one must be sure one is, observationally and theoretically, in the asymptotic region or, if not, make allowances for it. Thus, having stated ( $\$ 7)$ that ACRIM data indicate $\mathrm{P}_{0} \sim 37$ minutes, he then goes on to suggest ( $\S 8$ ) that nevertheless, averages of standard model period separations over just the first 10 or 20 modes would yield $\mathrm{P}_{\mathrm{O}} \sim 29$ or 32 minutes; should ACRIM results only be sensitive to these modes, claims for such low $\mathrm{P}_{\mathrm{O}}$ 's could still be consistent with standard models. This is a typically ingenious suggestion. But, if the formerly firm predictions of standard models are to give ground in this manner, the flexibility it indicates should also be accorded to the less macho WIMP predictions. Although the relevant calculations are not available, Cox's suggestion would equally imply that a measured $P_{0}$ as low as $\sim 23$ minutes could be consistent with the WIMP model.

In many searches for g-mode $\mathrm{P}_{\mathrm{o}}$ 's, too much adherence may have been made to theoretical predictions of expected ranges. Perhaps prompted by the mistaken belief that all solutions to the solar neutrino puzzle implied a $\mathrm{P}_{\mathrm{o}} \gtrsim 36-37$ minutes, searches have tended to commence from a lower limit only modestly below this value -- e.g. the previous ACRIM search for $\mathrm{P}_{\mathrm{O}} \geq 33.5$ minutes of Fröh1ich and Delache (1984). Since Fröh1ich's latest search involved the range $P_{0} \geq 25$ minutes, there is a danger, following Cox's suggestion, that history could repeat itself -- even this new range may still be too limited! My colleague Douglas Gough tells me that he has been pleading with observers for years to place less trust in theorists and to broaden their g-mode period horizons. Let us hope that in a not too distant future, his warning on the limitations of theoretical trustworthiness will really be heard!

\section{ACKNOWLEDGEMENTS}

I would like to express my appreciation to J. Christensen-Dalsgaard for his last-minute invitation to attend this meeting, and to acknowledge my debt to colleagues Ronald L. Gilliland, Mayank N. Vahia, and Douglas 0. Gough, from whom in particular I have no doubt failed to learn enough. This work was supported in part by the U.S. National Science Foundation 
through grant number AST 83-11406.

\section{REFERENCES}

Cleveland, B.T., Davis, R. and Rowley, J.K. 1984, in Resonance Ionization Spectroscopy 1984 (eds. Hurst, G.S. and Payne, M.G.; Institute of Physics Conf. Ser. 71, Bristo1), p. 241.

Faulkner, J. and Gilliland, R.L. 1985, Astrophys. J. 2999, 994.

Faulkner, J., Gough, D.0. and Vahia, M.N. 1986, Nature 32121, 226 .

Fröhlich, C. and Delache, P. 1984, in Solar Seismology from Space, JPL $\underline{\underline{84}} \underline{\underline{8}} \underline{\underline{4}} \underline{\underline{1}}$ p. 183 .

Iben, I. Jr. 1969, Ann. Phys. 폴, 164 .

Schatzman, E., Maeder, A., Angrand, F. and Glowinski, R. 1981, Astron. Astrophys. $\underline{\underline{96}}, 1$. 